treated with tannic acid jelly containing one part in 5,000 of merthiolate. Impaiment of liver function was least in the group treated with gentian violet and silver nitrate, although the burns in this group were much larger. In a large series of children treated with silver nitrate, there was no case of the acute toxæmia which may occur after tannic acid treatment. Impairment of liver function was commonest and most severe in the group treater with tannic acid. The authors think that this agrees with clinical experience. They suggest that the merthiolate in the tannic acid jelly used may have been partly responsible for the impairment of liver function.

Seven cases with injuries covering 5 per cent of the body surface were treated with sulphacetamide paste, and these occupied an intermediate position between the groups treated with tannic acid and gentian violet-silver nitrate. The authors point out that sulphonamide drugs may be rapidly absorbed from superficial burns and may also cause liver damage ; they should be used with care. Their general conclusion is that there is now sufficient clinical and experimental evidence to justify the abandonment of tannic acid as a local application for burns. R. H. Franklin (The Practitioner, 62, 167 ; 1944) also discusses the use of tannic acid and agrees that it is being largely abandoned now. Rae and Wilkinson think that coagulation treatment may save life in the early stages of very extensive superficial burns and that, from the point of view of toxic absorption, silver nitrate is probably a safe coagulant. In an annotation, The Lancet (March 11, 1944, p. 344) refers to other experimental work on the toxicity of tannic acid which has been done on dogs, rats and mice, and says: "There seems little doubt now about the experimental facts concerning the toxicity of tannic acid". The British Medical Journal (April 1) agrees and adds that, although mortality from burns has diminished since tannic acid was introduced in 1925, there is no evidence that it has "any advantage in this respect over other methods".

\section{Classification of Diseases and Injuries}

Asked by the Ministry of Health to advise on a system for collecting and recording statistics of patients admitted to hospital, the Medical Research Council appointed a Committee on Hospital Morbidity Statistics, which has now issued "A Provisional Classification of Diseases and Injuries for Use in Compiling Morbidity Statistics" (Med. Res. Council, Special Report Series, No. 248. H.M. Stationery Office, 1944. 3s. net). The classification here recommended has been adopted by the Ministry of Health for the classification of all Emergency Medical Service records, and the Ministry of Pensions is also using it. It will also be used in the Regional Bureaux of Health and Sickness records which the Nuffield Provincial Hospitals Trust is establishing in some areas. It is comparable with the International List of Causes of Death and the Diagnosis Code of the United States Public Health Service, the code numbers of both of which are included. It is also comparable with the Diagnosis Codes of the Royal Navy, Army and R.A.F. Medical Services. The coding system has been planned for use with mechanical sorting, but may be used equally well with manual filing. It is not suggested that it should replace the Standard Nomenclature of Disease of the American Medical Association.

The Committee has issued this provisional scheme as a Special Report so that it may be available for as wide a trial as possible. Dr. Percy Stocks, in a brief historical introduction, reminds us that every deceased person was either a sick or an injured person and, if his illness is classified during life, it will usually be the same as the cause of death defined in the Registrar-General's Manual. But the bulk of illnesses do not lead to death, so that a classification of diseases and injuries must give greater attention to minor and disabling conditions than a classification of causes of death needs to do. It is recognized that the diagnoses of some diseases, even when they are made by experts, may not agree, so that only tentative groupings are made in some sections. In some groups (for example, heart disease) the classification has been framed with the object of getting information necessary for a better classification. The Committee hopes, indeed, that any errors, omissions or inconsistencies observed will be reported, so that they may be incorporated in a future revised edition.

\section{Value of Human Milk}

BradFord-HrL has pointed out in his "Principles of Medical Statistics" that bottle-fed babies are 'selected', in that some factor has determined whether or not the mother feeds her baby. Deductions as to the relative values of breast- and bottle-feeding, based on comparisons of growth and health of groups of babies fed by one or other method, are vitiated by this selection. Mr. Eric Wood points out, in an article in the Medical Press and Circular of February 9 , that no controlled experiment has been made in which the two groups have been comparable at the start, in heredity, environment and physical conditions. Nor is this possible; for readiness to agree to give up breast-feeding would, in itself, make the 'bottle' group a selected one. In default of this possibility, Mr. Wood points out the need for much more information on the following points. (1) The effects of the mother's environment, food, etc., on the ability to suckle and on the quality and quantity of the milk. We now realize that the easy assumption that the mother is sacrificed to the baby does not hold without qualification. (2) The response of babies to their food. Is the flying start of the breast-baby due to the composition of the colostrum ? Babies are not, like lambs, dependent on the colostrum for a supply of antibodies from their mothers; antibodies (the antibody to the $R h$ factor, for example) pass through the human placenta. Both flying start and ability to suckle may both be evidence of a superior reproductive capacity. (3) The advantages, if any, of breast-fed babies that persist into later life. Mr. Wood points out that this information would be given only by long-term investigations based "On co-ordinated planning, a long time in advance, by some suitable 'ad hoc' committee or other body". Actually, very valuable work into the composition of human milk has been carried out, for two years, at the National Institute for Research in Dairying, supported by the Medical Research Council; and more extensive investigations, on the lines suggested, had been planned and work on them had started at the beginning of this year.

\section{President Jefferson, Statesman-Scientist}

THE recent bicentenary celebrations of Thomas Jefferson's birth have directed attention once again to the versatility of this remarkable man, some of whose special scientific interests are the subject of a 'pre-reprint' of 64 pp., with illustrations and maps, from Chronica Botanica (8, Nov. 1943), of a study by 